\title{
Salivary Gland Polymorphous Low Grade Adenocarcinoma
}

National Cancer Institute

\section{Source}

National Cancer Institute. Salivary Gland Polymorphous Low Grade Adenocarcinoma. NCI

Thesaurus. Code C35702.

A low grade malignant epithelial neoplasm arising from the salivary gland. It usually arises from the oral cavity, with approximately $60 \%$ of the cases involving the palate. The most common presenting sign is a painless mass in the palate. It is characterized by the presence of uniform, small to medium size malignant epithelial cells and an infiltrating pattern. There is a variety of morphologic patterns which may be present within an individual tumor, including lobular, papillary, papillary-cystic, cribriform, and trabecular pattern. It usually has a favorable clinical outcome and a low metastatic potential. 